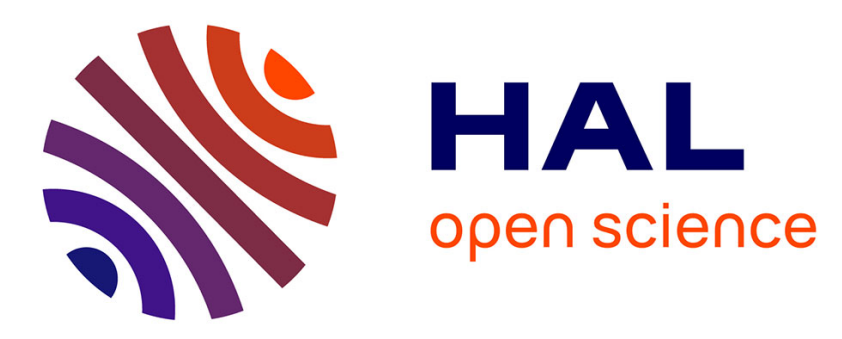

\title{
Alterations of EEG rhythms during motor preparation following awake brain surgery
}

\author{
Anthony Boyer, Sofiane Ramdani, Hugues Duffau, Bénédicte \\ Poulin-Charronnat, David Guiraud, François Bonnetblanc
}

\section{To cite this version:}

Anthony Boyer, Sofiane Ramdani, Hugues Duffau, Bénédicte Poulin-Charronnat, David Guiraud, et al. Alterations of EEG rhythms during motor preparation following awake brain surgery. Brain and Cognition, 2018, 125, pp.45-52. 10.1016/j.bandc.2018.05.010 . hal-01813291

\section{HAL Id: hal-01813291 \\ https://hal.science/hal-01813291}

Submitted on 12 Jun 2018

HAL is a multi-disciplinary open access archive for the deposit and dissemination of scientific research documents, whether they are published or not. The documents may come from teaching and research institutions in France or abroad, or from public or private research centers.
L'archive ouverte pluridisciplinaire HAL, est destinée au dépôt et à la diffusion de documents scientifiques de niveau recherche, publiés ou non, émanant des établissements d'enseignement et de recherche français ou étrangers, des laboratoires publics ou privés. 


\section{Alterations of EEG rhythms during motor preparation following awake brain surgery}

Anthony Boyer $a$, Sofiane Ramdani $b$, Hugues Duffau $c, d$, Bénédicte Poulin-Charronnat $e$, David Guiraud $a$, François Bonnetblanc $a, f, g$

a

CAMIN team, INRIA, LIRMM, Université de Montpellier, 34095 Montpellier, France.

b

IDH team, LIRMM, Université de Montpellier, 34095 Montpellier, France.

c

"Plasticity of Central Nervous System, Stem Cells and Glial Tumours", INSERM

U1051, Institut of Neurosciences of Montpellier, 34295 Montpellier, France.

d

Département de Neurochirurgie, Centre Hospitalier Régional Universitaire de

Montpellier Gui de Chauliac, 34295 Montpellier, France.

e

LEAD, CNRS UMR5022, Université Bourgogne Franche-Comté, 21000 Dijon, France.

$\mathbf{f}$

"Cognition, Action et Plasticité Sensorimotrice", INSERM U1093, UFR STAPS,

Université Bourgogne Franche-Comté, 21078 Dijon, France.

g

Institut Universitaire de France, 75231 Paris, France.

Corresponding author: francois.bonnetblanc@inria.fr 


\begin{abstract}
Slow-growing, infiltrative brain tumours may modify the electrophysiological balance between the two hemispheres. To determine whether and how asymmetry of EEG rhythms during motor preparation might occur following "awake brain surgery" for this type of tumour, we recorded electroencephalograms during a simple visuo-manual reaction time paradigm performed by the patients between 3 and 12 months after surgery and compared them to a control group of 8 healthy subjects. Frequency analyses revealed imbalances between the injured and healthy hemispheres. More particularly, we observed a power increase in the $\delta$ frequency band near the lesion site and a power increase in the $\alpha$ and $\beta$ frequency bands. Interestingly, these alterations seem to decrease for the two patients whose surgery were anterior to 9 months, independently of the size of the lesion. Reaction times did not reflect this pattern as they were clearly not inversely related to the anteriority of the surgery. Electrophysiology suggests here different processes of recovery compared to behavioral data and brings further insights for the understanding of EEG rhythms that should not be systematically confounded or assimilated with cognitive performances. EEG monitoring is rare for these patients, especially after awake brain surgery, however it is important.
\end{abstract}

Key words: diffuse low-grade glioma - awake brain surgery - spectral analysis Brainwaves

\title{
Highlights:
}

- Awake brain surgery alters spectral content of EEG signal.

- Brain electrophysiological activity seems to normalize over time.

- By contrast, reaction times are not related to the anteriority of the surgery. 


\section{Introduction}

Low-Grade Gliomas (LGG - World Health Organization grade II gliomas) are slow-growing primary brain tumours that develop at the expense of glial cells. Because of their infiltrative and diffuse nature, maximizing the resection of tumoural tissue bordering or merging with functional areas brings important risks of neurological impairments (Duffau and Capelle 2004). It is therefore crucial to distinguish the eloquent cortex and preserve structures supporting essential cognitive processes such as language or motor control from surgical injury (Plaza et al. 2009; Schucht et al. 2013). In this context, direct electrical stimulation (DES) is used during wide-awake brain surgery for online mapping of brain functions (Duffau 2015, Mandonnet et al. 2010). The neurosurgeon applies DES with a bipolar stimulator on the surface of the brain and along underlying white matter tracts while the patient is asked to perform a set of motor, language, and cognitive tasks. If the patient consistently fails neuropsychological tests during the stimulation, this means that the region remains functional despite the invasion of the lesion and should be preserved. Conversely, if the stimulated region does not cause deficits in appropriate tasks, this means that its function is taken over by another region. This surgical routine allows the progressive identification and optimal resection of the tumour up to the functional limits (Duffau 2015).

Tumour discovery mostly relies on first epileptic seizure or incidental detection rather than neurological complaints from patients. It has been suggested that the slow progression of LGG contrasts with more acute lesions, allowing the central nervous system to ensure functional homoeostasis while the tumour disseminates to other brain areas (Desmurget et al. 2007; Duffau 2013). This very unique physiopathological model and associated neuroplasticity phenomena were investigated in several works, which have suggested various patterns of functional redistribution with progressive involvement of both ipsi- and contralesional areas in the compensation of gradually damaged neuronal circuits (Bonnetblanc, et al. 2006; Duffau 2014a; Duffau 2014b).

After surgical resection, patients only present transient impairments despite massive exeresis volumes, and recover within 3 months showing no clinical deficits in almost all cases. These deficits are hardly detectable using classical neuropsychological evaluations (Duffau et al. 2003; Herbet et al. 2013). Regarding, visuo-spatial and motor cognition, preliminary studies proposed dedicated paradigms for the assessment of recovery kinetics. Sallard et al. (2012a) and Charras et al. (2014) both used standard line bisection tests to reveal transient hemispatial neglect and neglect-like symptoms in the acute post-operative phase preceding fast and substantial recovery in all patients. Sallard et al. (2012b) also proposed a visuo-manual reaction time paradigm to evidence longer reaction times for the contralesional hand. These results clearly suggest some degree of functional asymmetry during the postoperative period, and were hypothesized to represent more global physiological and functional imbalance between brain hemispheres, including subcortical structures (e.g. Thalamus and cerebellum), induced by tumour resection (Boyer et al. 2015a; Boyer et al. 2015b).

Apart from behavioural aspects, little is known regarding the consequences of awake brain surgery on postoperative brain dynamics. In order to clarify the role of each hemisphere in compensation processes and possible electrophysiological imbalances, Bonnetblanc et al. (2014) measured the electroencephalographic (EEG) activity of 5 patients performing an 
analogous visuo-manual reaction time task between 3 and 12 months after surgery. The experiment consisted of combinations of visual stimulus location and responding hand to supposedly recruit each hemisphere to a different extent. The study focused on the amplitude of event-related potentials (ERPs) to probe asymmetries of cortical excitability. Surprisingly, they found that excitability was increased in the injured hemisphere but for patients with the smallest lesions and independently of the anteriority of the surgery. Neither behavioral scores nor excitability changes were illustrative of the time passed from the operation.

In the present work, we complement these investigations focusing on the motor preparation period preceding the hand response to study the stationary EEG signal before the ERP and further document the consequences of tumour resection and functional reorganisation on brain electrophysiological dynamics.

We performed usual spectral analyses and searched for potential differences in the amplitude and spatial distribution of common "Brainwaves". These rhythms typically refer to oscillatory activities recorded over the scalp and commonly grouped into bands depending on their frequency, amplitude, and location (Arroyo et al. 1993; Buzsaki and Draguhn 2004; GarciaRill et al. 2016). Their physiological origins and functional role are still highly debated but emerging evidence from animal and human studies suggests their importance in brain spatiotemporal integration and segregation (Varela et al. 2001). Modifications of brain oscillation patterns have been associated with diseases of the central nervous system and quantitatively assessed in EEG studies as clinical indicators, especially in the context of cognitive disorders, seizure, and more particularly, ischemic strokes (Harpale and Bairagi 2016; Medeiros Kanda et al. 2009; Rabiller et al. 2015). However, alterations of brainwaves in the presence of a brain tumour remain particularly unclear, leading to inconsistent results even though a few MEG and EEG studies highlighted an overall slowdown in electrophysiological activity of the brain, i.e. increased spectral power in the $\delta(1-4 \mathrm{~Hz})$ and $\theta(4-8 \mathrm{~Hz})$ frequency ranges (Decker and Knott 1972; Jongh et al. 2003; Preuß et al. 2015; Selvam and Shenbagadevi 2011; Selvam and Devi 2015; Wijk et al. 2012). None of these studies focused on brain oscillations after awake brain surgery and, to the best of our knowledge, no study confirmed the presence of slow waves after the tumour removal. From a functional perspective, the attentional task preceding motor response offers a constrained environment similar to motor preparation paradigm as seen in Brain Computer Interface studies (Han and Bin 2014) which may help decipher potential modifications of induced brain oscillatory activity, contrasting with ambiguous resting states EEG studies. Therefore, we placed special emphasis on $\alpha$ rhythms (9-12Hz) and sensorimotor rhythms (consisting of $\mu: 9-12 \mathrm{~Hz}$ and $\beta: 14-20 \mathrm{~Hz}$ bands), potentially emerging in occipital and Rolandic areas respectively. These particular oscillations have been linked to visual processing, motor control, motor imagery, action anticipation, and are therefore likely to be elicited during the motor preparation period (Arroyo et al. 1993; Denis et al. 2017; Han and Bin 2014; Heinrichs-Graham et al. 2017; Kim and Kim 2016; Sabate et al. 2012). The perirolandic region is of particular interest because of its low interindividual variability (Mellerio et al. 2016). It contains sensorimotor areas which are direct input/oupout for sensory and motor systems and are thus unlikely to be compensated in other locations (Herbet et al. 2016).

Using spectral analyses, we aim to study the EEG signal during motor preparation period in the frequency domain to provide complementary information and new insights regarding the 
consequences of awake surgery on brain electrophysiological activity.

\section{Materials \& Methods}

\subsection{Participants}

\begin{tabular}{|c|c|c|c|c|c|c|c|c|}
\hline \multicolumn{5}{|c|}{ Socio-demographic } & \multicolumn{3}{|l|}{ Clinical } & \multirow{2}{*}{$\begin{array}{l}\text { Time of assessment afte } \\
\text { surgery (months) }\end{array}$} \\
\hline Id. & $\begin{array}{l}\text { Age } \\
\text { (years) }\end{array}$ & Gender & $\begin{array}{l}\text { Edu. level } \\
\text { (years) }\end{array}$ & Handedness & $\begin{array}{l}\text { Grade of } \\
\text { the tumour }\end{array}$ & Surgery & $\begin{array}{l}\text { Volume of } \\
\text { resection } \\
\text { (cm3) }\end{array}$ & \\
\hline P1 & 35 & $M$ & 17 & $\mathrm{R}$ & II & Awake & 7.1 & 3 \\
\hline P2 & 38 & $M$ & 15 & $\mathrm{R}$ & II & Awake & 79.2 & 3 \\
\hline P3 & 44 & $F$ & 12 & $R$ & II & Awake & 119.1 & 3 \\
\hline P4 & 33 & $\mathrm{M}$ & 17 & $\bar{R}$ & II & Awake & 116.8 & 9 \\
\hline P5 & 45 & $M$ & 17 & $\mathrm{R}$ & II & Awake & 11.5 & 12 \\
\hline
\end{tabular}

Table 1: Patient information: Socio-demographic and clinical data of the 5 patients enrolled in this study.

(a) Patient 1

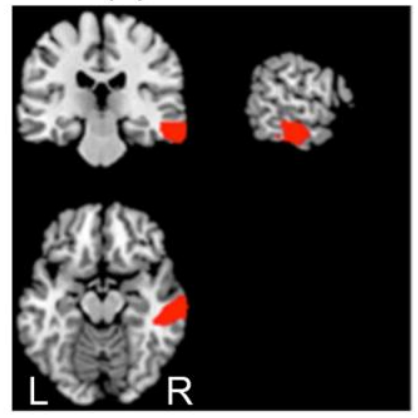

(d) Patient 4

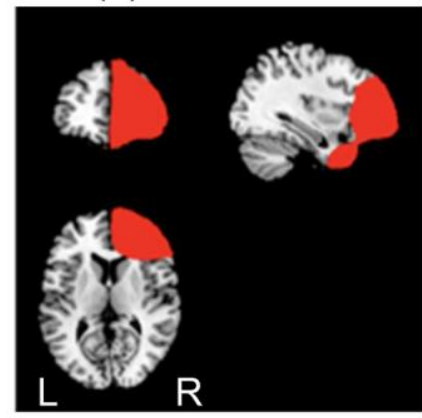

(b) Patient 2

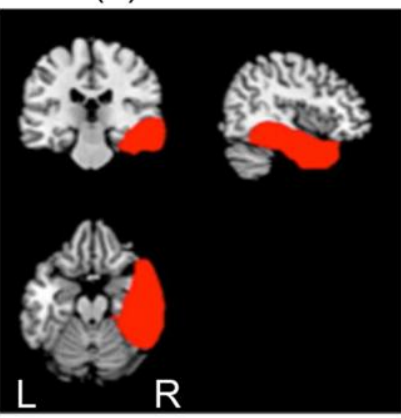

(e) Patient 5

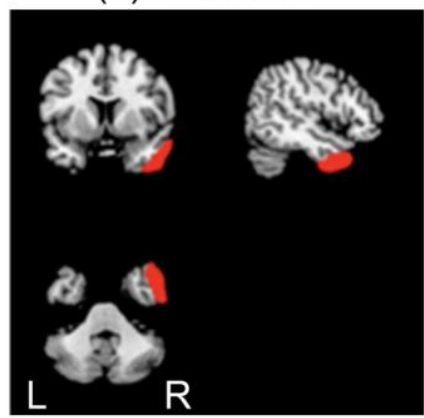

(c) Patient 3

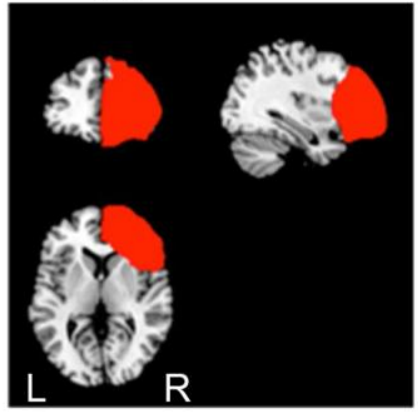

\section{.}

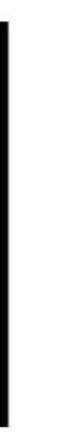

Figure 1: Lesion mapping: Result of the lesion mapping performed 3 months after surgery for each patient.

Five right-handed patients (P1 to P5; 4 males and 1 female; mean \pm SD age, $39 \pm 5.34$, range $=[33-45])$ and eight age-matched, right-handed, healthy controls ( 7 males and 1 female; mean \pm SD age, $39.75 \pm 4.30$, range $=[33-45])$ participated in the study. None of the controls had a history of neurological disease (including motor, visual hemifield, or visuo-spatial impairments). The study complied with the World Medical Association's Code of Ethics (the 1964 Declaration of Helsinki and its amendments). The patients' socio-demographic and 
clinical characteristics are summarized in table 1.

P1 (a 35-year-old male) presented with a tumour at right middle and inferior temporal area (Fig.1a). P2 (a 38-year-old male) presented with a right temporal tumour (Fig.1b). P3 and P4 (a 44-year-old female and a 33-year-old male, respectively) presented with a right frontal tumour (Fig.1c, Fig.1d). P5 (a 45-year-old male) presented with a tumour in the right temporal pole (Fig.1e). All patients underwent the surgical resection of LGG (grade II, according to the World Health Organization classification) with intra-operative DES.

Lesion mapping was based on whole-brain three-dimensional T1 anatomical MRI data sets acquired 3 months after surgery (on a 3T Siemens Skyra machine, Siemens Medical Systems, Erlangen, Germany). These individual MRI data sets were then normalized against the Montreal Neurological Institute template by applying cost function masking (Brett et al. 2001). This method has been well characterized and is known to be the best option for normalization when the lesion is extensive (Andersen, Rapcsak, and Beeson 2010). During the registration process, cost function masking avoids the bias caused by abnormal, lesioninduced, radiological signals. Briefly, the lesion was contoured (using MRIcron software (http://www.mccauslandcenter.sc.edu/mricro/mricron) (Rorden, Karnath, and Bonilha 2007) and transformed into a binarized image. This image was inserted during the normalization $\begin{array}{llllll}\text { processes } & \text { performed } & \text { here } & \text { with } & \text { SPM } & 8\end{array}$ (http://www.fil.ion.ucl.ac.uk/spm/software/spm8)(Ashburner 2012). The lesion is then redrawn on the normalized scan, in order to generate an individual volume of interest.

Patients were thoroughly examined by a neuropsychologist before and after surgery. None had clinically evident neurological disorders. Data on surgical resection volumes are summarized in table 1. Three patients had extensive resections $\left(79.2,119.1\right.$ and $\left.116.8 \mathrm{~cm}^{3}\right)$, and two had much smaller resections $\left(7.1\right.$ and $\left.11.5 \mathrm{~cm}^{3}\right)$. P3 was undergoing her second surgical operation on the frontal lobe. The first operation had been performed in 2004 by another neurosurgeon, but the lesion had continued to grow in the intervening time.

\subsection{The behavioural task}

Participants comfortably sat in front of a laptop computer on which different visual stimuli (including the "go" signal) were displayed with Presentation ${ }^{\circledR}$ software (http://www.neurobs.com). The screen was about $60 \mathrm{~cm}$ from the body in the sagittal axis and $45 \mathrm{~cm}$ below eye level. Two switches were positioned on each side of the sagittal axis $(15 \mathrm{~cm}$ apart and $45 \mathrm{~cm}$ from the body).

The motor responses consisted of pressing one or both switches with the corresponding index finger(s). A fixation cross appeared in the middle of the screen and served as the warning signal. After a variable time interval, one or two arrows appeared at different locations (represented the "go" signal); the participant sawing one or two arrows then had to press the corresponding button(s), as soon as possible. The arrow(s) could appear to the left of the initial fixation cross (designated as LS), to the right (RS), to both the left and the right at the same time $(\mathrm{R}+\mathrm{LS})$ or at the fixation cross's central position (CS).

The time interval between the appearance of the fixation cross and the appearance of the "go" signal (i.e. the stimulus onset asynchrony) varied randomly from one trial to another in the range 1'000 to 2'000 ms. This interval comprised two phases. Firstly, the fixation cross was displayed for a duration between 1'000 and 1'500 ms. After the disappearance of fixation 
cross, the stimulus appeared after a variable interval of between 0 and $500 \mathrm{~ms}$. The four combinations of visual stimuli (RS, LS, R + LS, and CS) were randomized within three blocks that depended on the hand(s) used (i.e. RH, LH, and R $+\mathrm{LH}$ ), resulting in twelve conditions. A total of 360 trials (30 trials $\times 12$ conditions) were performed in two separate sessions.

\subsection{EEG recording and pre-processing}

The electroencephalogram was recorded at 64 scalp sites using a 10-20 system (BioSemi ActiveTwo). Horizontal eye movements were recorded with electrodes placed on the outer left and right canthi. Vertical eye movements were recorded with an electrode placed under the right eye. Two additional electrodes were placed on the left and right mastoids (A1, A2). During recording, the BioSemi system's common mode sense electrode served as the reference electrode. Electrophysiological signals were digitized at a 2'048 Hz sampling rate and acquired with ActiView software. The EEG signals were analysed using the EEGLAB toolbox (https://sccn.ucsd.edu/wiki/EEGLAB) (Delorme and Makeig 2004) for Matlab (MATLAB and Statistics Toolbox Release 2012a, The MathWorks, Inc., Natick, Massachusetts, United States). The data were re-referenced against the mean of electrodes A1 and A2, down sampled to $250 \mathrm{~Hz}$ and filtered using a high-pass FIR filter with a cut-off frequency of $1 \mathrm{~Hz}$. In order to correct for eye-movement artefacts, EEGLAB's binica routine was used to perform independent component analyses by removing the first component. All the corrections underwent visual inspection. Eventually, we focused on motor preparation period restricting epochs to $1 \mathrm{~s}$ latency window going from -1 '000 $\mathrm{ms}$ prestimulus to the stimulus onset. In this particular context, only the condition related to the hand used remains relevant and the random combinations of visual stimuli were therefore pooled.

\subsection{Spectral analysis}

Power spectral densities (PSDs) were computed per epoch using Welch's modified periodogram estimation method (250 data points; 100 data point Hanning-window; 75\% overlap), producing an effective power value for each $1-\mathrm{Hz}$ iteration. For each subject and channel, resulting power spectra were averaged over all 120 trials according to the hand used (30 trials for each of the 4 randomized visual stimulus) and across the $1-40 \mathrm{~Hz}$ frequency range to cover commonly investigated Brainwaves $(\delta: 1-4 \mathrm{~Hz} ; \theta: 4-8 \mathrm{~Hz}$; occipital $\alpha$, rolandic $\mu$, temporal $\tau$ : 8-12 Hz; $\beta$ : $12-30 \mathrm{~Hz}$ and $\gamma: 30+\mathrm{Hz}$ bands) (Arroyo et al. 1993; Buzsaki and Draguhn 2004; Garcia-Rill et al. 2016).

Spectral data were also used to compute topographical maps of the spectral power distribution over the scalp, "PSD maps", extracting the mean power contained within aforementioned frequency bands. For visualization purposes, power spectra computed from epochs were first log-transformed before being averaged over trials and/or subjects. For some patients and healthy subjects, visual inspection of PSD maps clearly revealed that up to several electrodes located at the edge of the EEG headcap had artefactual data, typically due to inadequate contact between electrodes and the scalp. For this reason we chose to exclude the outermost electrode strip from our analyses. 


\subsection{Statistical analysis}

Reaction times, effective power values (over the 1-40 Hz frequency range) and mean power values (within bands of interest), for each patient, at each electrode, were compared with their respective $95 \%$ confidence interval estimated from the control group. Comparisons were performed for each hand condition. The smallness of the control group limits statistical inferences regarding underlying probability distribution of the data. In this context, confidence intervals were estimated using a resampling technique, i.e. Bootstrapping technique, applying the Bias Corrected and Accelerated percentile (BCA) method. The sampling distribution of statistical means of each variable (reaction times and power values) was computed using 1'000 bootstrap samples and the confidence interval was deduced from the estimated distribution (Carpenter and Bithell 2000).

\section{Results}

\subsection{Spectral analysis}

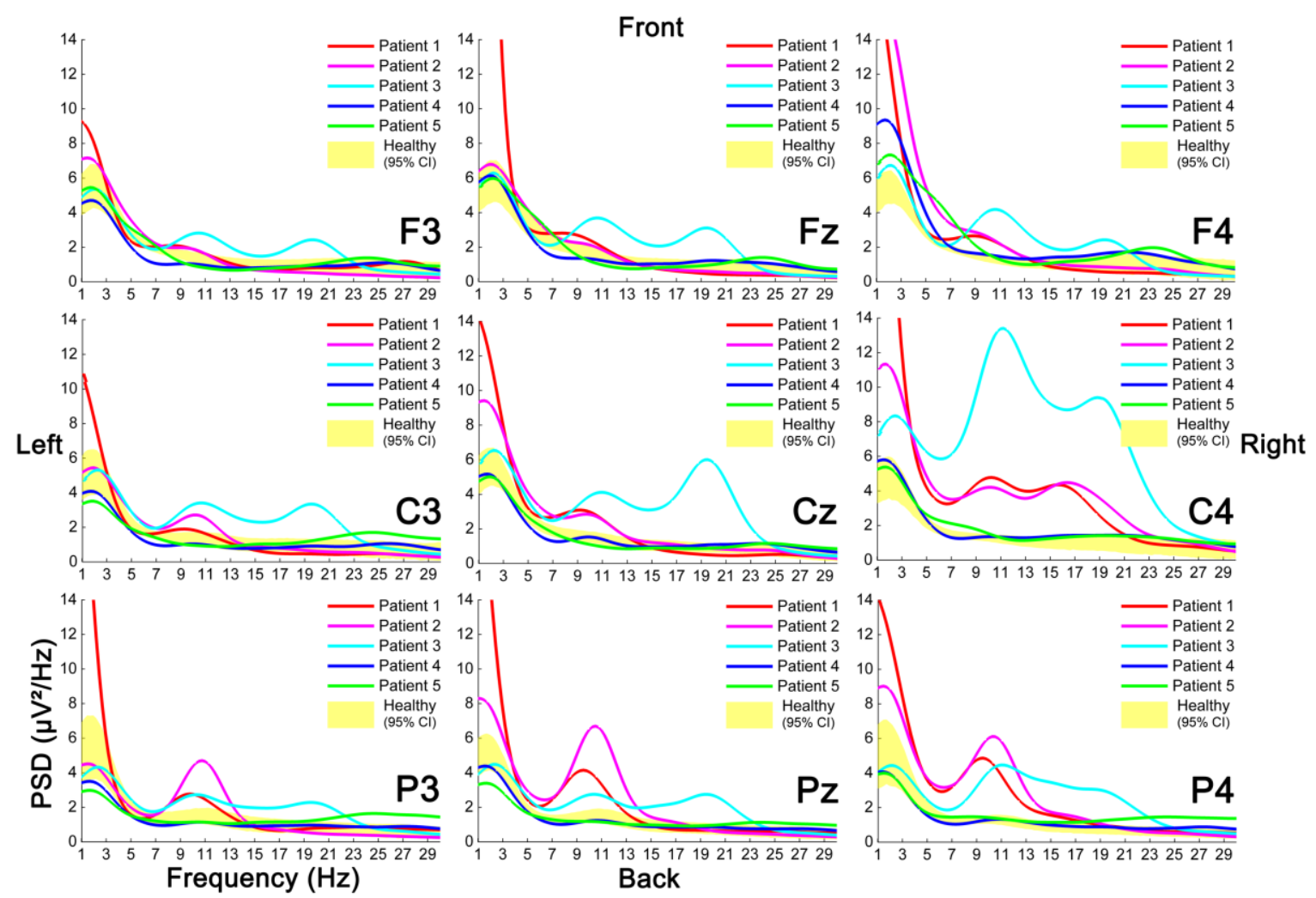

Figure 2: Power spectral densities: Example of PSDs we obtained for patients (single lines) and associated $95 \%$ confidence interval estimated from the control group (yellow surface), for a subset of electrodes (F3, Fz, F4; C3, Cz, C4; P3, Pz, P4). Results for the left hand (LH) condition are presented here. 


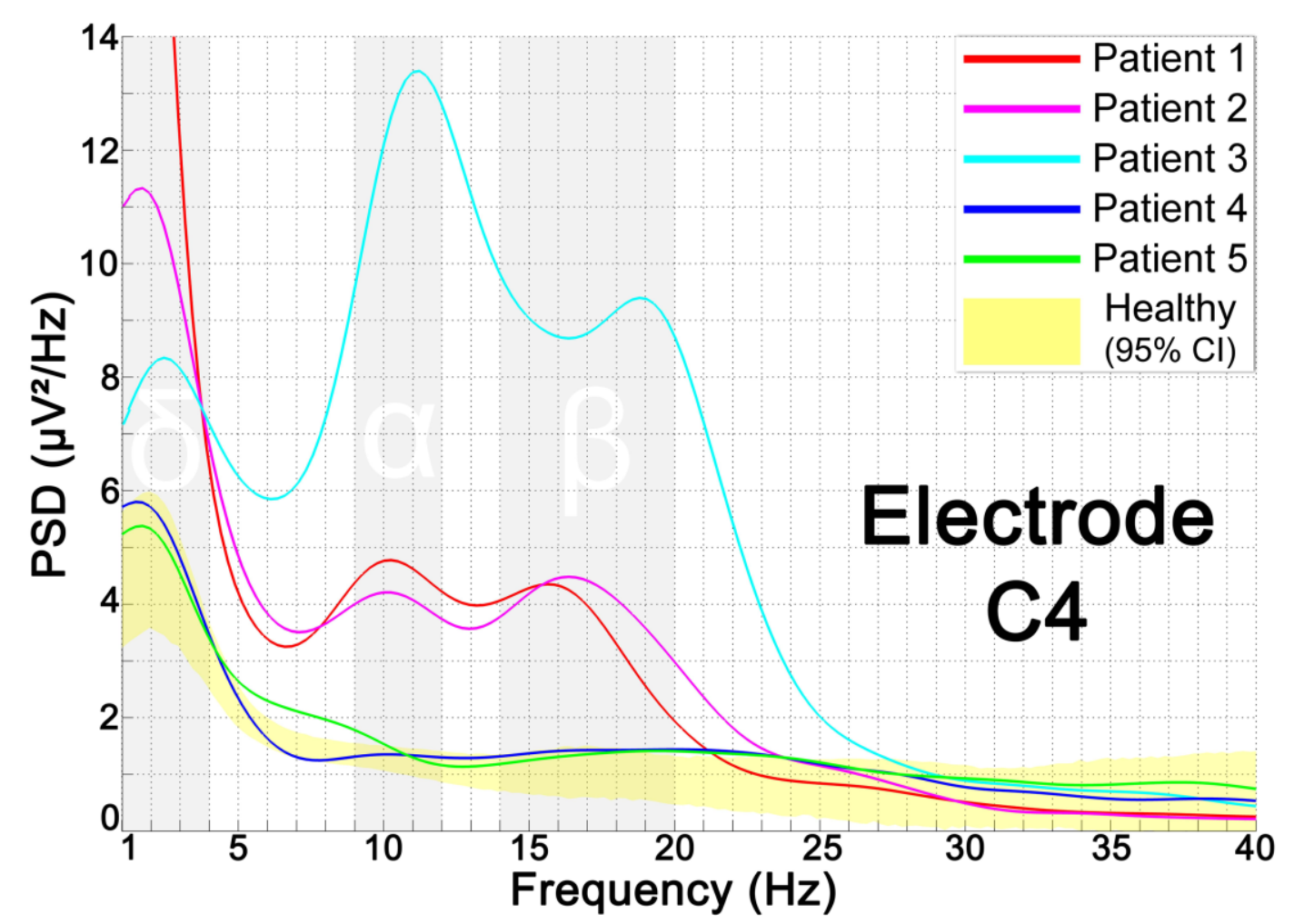

Figure 3: Power spectral densities at C4: PSDs for patients (single lines) and associated 95\% confidence interval estimated from the control group (yellow surface) at the electrode $\mathrm{C} 4$.

Results for the left hand (LH) condition are presented here.

Among the 5 patients enrolled in this study, 3 showed substantial alterations of oscillatory activity in comparison with the healthy group. The 3 patients were those who followed the experimental protocol the soonest after wide-awake surgery, namely P1, P2 and P3, who were recruited 3 months after tumour resection. The 2 other patients, P4 and P5, followed the experimental protocol respectively 9 and 12 months after surgery and showed marginal, barely significant, alterations of spectral content. As seen in figure 2 and figure 3 , the major differences arise within $\delta, \alpha$ and $\beta$ bands with significantly higher spectral power and multiple peaks, which are not seen in healthy subject spectra. PSD maps, illustrated in figure 4, revealed that higher power mainly occurs within the injured (right) hemisphere, contrasting with a more balanced distribution of spectral densities for the control group.

Similarly to reaction times (see below), the hand used did not yield any notable differences regarding spectral content, therefore we chose to present only the results associated with the Left Hand (LH) condition.

Increase in $\delta$ activity: $\delta$ power appeared significantly higher for all 5 patients compared to healthy subjects, especially when moving closer to the lesion site. P3 and P4, both with a right frontal tumour, showed maximum $\delta$ activity in superior frontal regions whereas P2 and P5, both a with right temporal lesion, showed highest scores in more lateral, fronto-temporal regions. This is however not the case of $\mathrm{P} 1$ with enhanced $\delta$ activity scattered over the cortex. Regarding P1, P2 and P3, $\delta$ power is higher over most of the scalp with respect to the control group. On the other hand, $\mathrm{P} 4$ and more particularly $\mathrm{P} 5$ showed more contrasted variations of $\delta$ 
activity with increased power near the lesion site and decreased power for some electrodes in contralesional parieto-occipital regions.

Increase in $\alpha$ activity: P1, P2 and P3 exhibited increased $\alpha$ power at almost all electrodes. For P1 and P2, maximum $\alpha$ activity was recorded within the occipital lobe, with an extension to ipsilesional somatomotor regions. For P3, $\alpha$ activity was mostly concentrated in ipsilesional peri-rolandic areas. P4 and P5, for their part, showed scarcely significant variations of $\alpha$ power in comparison with the healthy group. For theses patients, higher power arise in similar regions to those observed when studying spatial repartition of $\delta$ activity, i.e. close to the lesion site. However, a slight diminution of $\alpha$ power can be observed for a few electrodes of the contralesional frontal lobe for both patients.

Increase in $\boldsymbol{\beta}$ activity: $\mathrm{P} 1, \mathrm{P} 2$ and $\mathrm{P} 3$ showed highest $\beta$ activity around somatomotor regions, just below electrode $\mathrm{C} 4$ (figure 3 ). $\mathrm{P} 3$ was the only patient who presented a global increase of $\beta$ power across all scalp electrodes. P1 and P2 both exhibited diminished $\beta$ activity for a few electrodes of the contralesional frontal lobe. P4 and P5 showed more balanced $\beta$ activity with minor gain of power in similar regions to those observed when studying $\delta$ and $\alpha$ activity and in parieto-occipital regions for P5. It should be noted that power spectra computed for electrode $\mathrm{C} 4$ revealed observable differences of $\beta$ power depending on the hand used. $\mathrm{P} 1$ and $\mathrm{P} 2$ showed decreased $\beta$ activity for this particular electrode when the right hand is used in comparison with the 2 other conditions ( $\mathrm{LH}$ and $\mathrm{L}+\mathrm{RH}$ ).

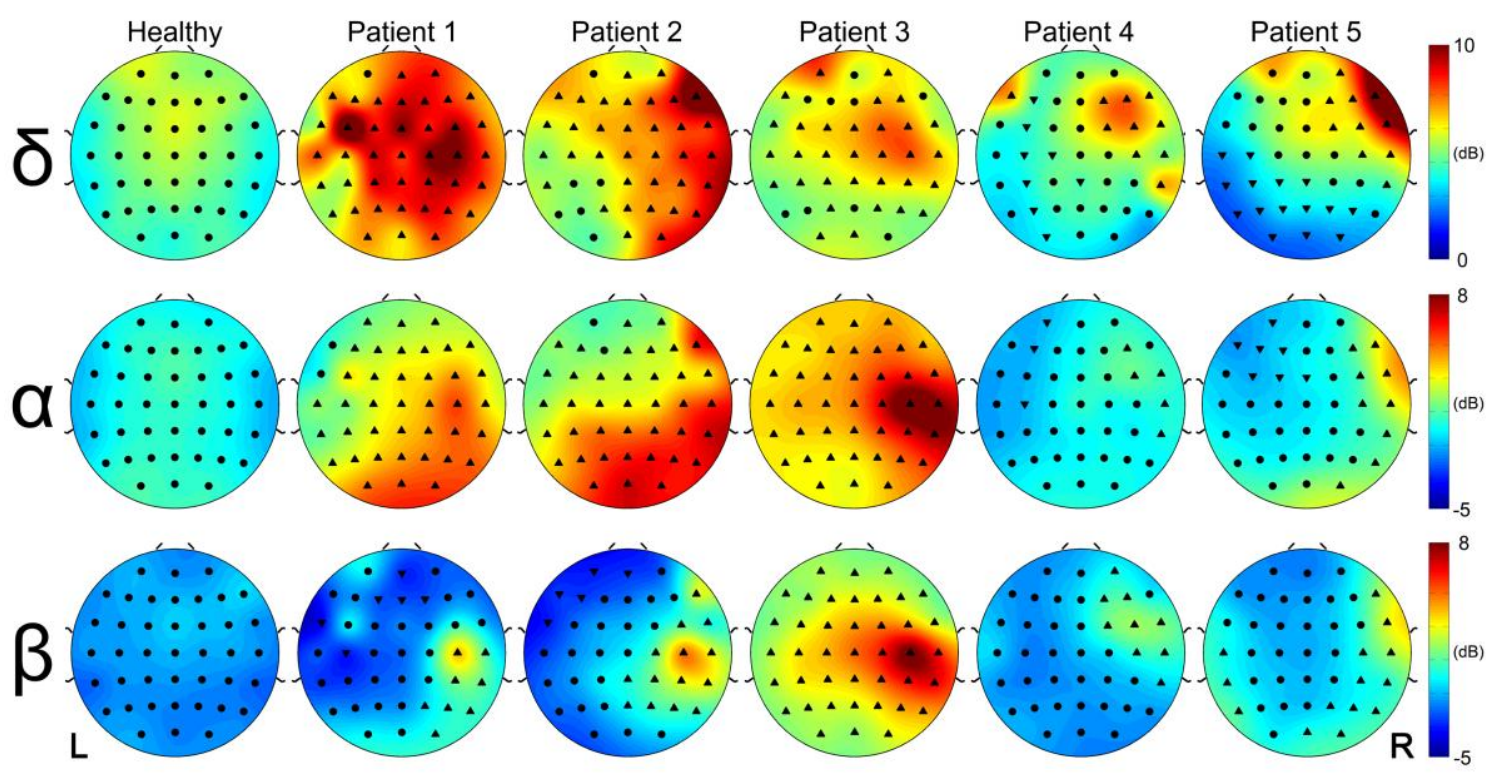

Figure 4: Topographical maps of spectral power: Maps illustrating the spatial distribution of the spectral power contained in a given frequency band over the scalp. We selected bands which showed significant peaks of spectral power for patients in comparison with the control group (i.e. $\delta$ band: $1-4 \mathrm{~Hz}, \alpha$ band: $9-12 \mathrm{~Hz}, \beta$ band: $14-20 \mathrm{~Hz}$ ), and we then calculated the average power at each electrode for each band. Electrodes are represented by a set of symbols: • symbol is used when the corresponding spectral power falls within the $95 \%$ confidence interval estimated from healthy subjects. $\boldsymbol{\Delta}$ and $\boldsymbol{\nabla}$ symbols are respectively used when the power is either greater or smaller than the $95 \%$ confidence interval. Results for the left hand $(\mathrm{LH})$ condition are presented here. 


\subsection{Reaction time analysis}

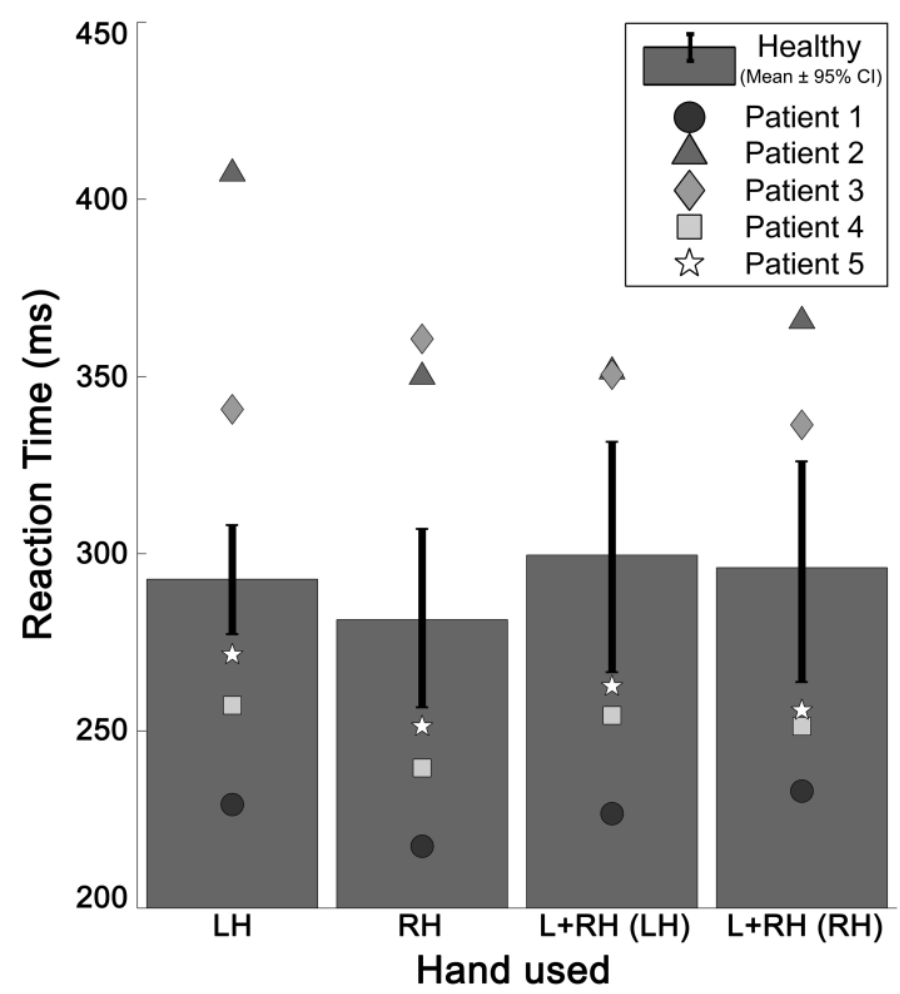

Figure 5: Reaction times: Reaction times for the control group (histograms: means \pm 95\% CI) and patients (geometric forms: individual means) depending on the hand used. CI - confidence interval, LH - left hand, RH - right hand.

As shown in Fig.5, P2 and P3 (with extensive right temporal and frontal lobe resection, respectively) showed significantly extended reaction times in all 3 conditions. On the other hand, P4 exhibited shorter reaction times although he also underwent an extensive resection of the right frontal lobe. Similarly, patients with smaller lesions, P1 and P5, showed abnormally diminished reaction times.

Despite substantial differences between patients and the control group, no significant differences in reactions times were identified when considering the hand used during the experimental protocol. These results did not mirror those obtained for spectral analyses. The size of the lesion can be large but the reaction times smaller (see P4). By contrast the normalization of spectrum can appear after 9 months despite very different lesion sizes (see $\mathrm{P} 4$ and P5), and $\delta$ band activities can be important despite a smaller lesion (see P1).

\section{Discussion}

In this study, we focused on postoperative brain dynamics of patients who underwent wideawake surgery for LGG. We analysed the EEG data of 5 patients, who performed a simple visuo-manual task, and compared them to a control group of 8 healthy subjects. We used the motor preparation period to extract power features to better characterise changes in EEG signal following surgery and subsequent functional reorganisation. We used spectral analysis to identify alterations of brainwaves over the cortex. Brainwaves have been investigated in 
various psychiatric and neurological diseases (Claus et al. 1998; Medeiros Kanda et al. 2009; Morita, Kamei, and Mizutani 2011; Swisher 2017) as well as brain injuries (Pevzner et al. 2016; Rabiller et al. 2015), but because of the heterogeneity of pathophysiological models and inter-individual variability, a general agreement regarding alterations of brain oscillatory rhythms is hard to reach. Nonetheless, several EEG studies helped identifying robust parameters discriminating patients and controls relying on a relatively strong dissociation between increased slower waves ( $\delta$ and $\theta$ ) and weakened faster activities ( $\alpha$ and $\beta$ ) (Brenner et al. 1988; Claus et al. 1998; Coben, Danziger, and Storandt 1985;; Forstl et al. 1996; Jongh et al. 2003; Koberda et al. 2013; Morita et al. 2011; Rabiller et al. 2015; Soininen et al. 1991). Neuronal origins for the slow down of EEG activity in the impaired brain are not fully understood but previous works suggested it to be linked with altered cerebral blood flow and cortical metabolism (Buchan et al. 1997; Foreman and Claassen 2012; Gueguen et al. 1991; O'Gorman et al. 2013; Szelies et al. 1997). More particularly, stroke studies proposed that the reduction of cerebral blood flow causes energy deprivation of pyramidal neurons, inducing changes in membrane potentials, leading to the slowdown of EEG activity (Rabiller et al. 2015). In this context, observed increases in $\delta$ waves we noticed for patients may be seen as an indication for altered metabolic activity as it appears to be roughly distributed within the injured side and maximized nearby the lesion site.

Awake brain surgery may induce neural activity depression concomitant with blood flow reduction in remote deafferented regions also known as diaschisis phenomenon (Boyer et al. 2015a; Boyer et al. 2015b), as well as more local disruption of perfusion balance. This imbalance might fade over time as patients who followed the experimental protocol 9 and 12 months after surgery showed limited alterations of $\delta$ activity. Even if the slow down of EEG activity is often associated with altered metabolism in stroke model and used as a predictive biomarker for post-stroke outcomes, $\delta$ waves were also considered in neuronal plasticity mechanisms and more precisely long-term potentiation (Assenza et al. 2015), and axonal sprouting (Carmichael and Chesselet 2002). Just as in stroke, overall power tends to be higher in both hemispheres with respect to healthy subjects especially when considering slow waves. It was suggested that acute deafferentation increased neural synchronization with new corticocortical junctions and enhanced neuronal excitability (Tecchio et al. 2005). ERP analysis previously performed on these data revealed concurring results with asymmetric interhemispheric excitability for patients (Bonnetblanc et al. 2014). In this context, origins for enhanced $\delta$ waves for patients who underwent wide-awake surgery are hard to disentangle and may be ascribed to metabolic disruptions and/or neuroplastic recovery. A few studies investigating wave abnormalities in the presence of a brain tumour (without surgery), revealed similar results with a slowing of background rhythms (Jongh et al. 2003; Selvam and Devi 2015; Wijk et al. 2012).

Less-expected result was the strengthening of faster activities with global increase in $\alpha$ power and cluster of $\beta$ activity near somatomotor regions for the 3 patients who were the most recently surgically operated at the time of the study. $\alpha$ waves are historically known to occur within the occipital lobe during wakeful relaxation with closed eyes and have been thought to reflect idling or inhibition of task-irrelevant cortical areas (Berger 1929; Palva and Palva 2007). Garcia et al. (2016) described $\alpha$ oscillations as resting rhythms, acting as a fulcrum for transitional brain states (Garcia-Rill et al. 2016). A point of contention is whether this 
frequency serves to process information relevant to the task at hand or rather to filter out irrelevant information and if it is generated by the neocortex, the thalamus, or a combination of both (Hughes and Crunelli 2005; Silva, Amitai, and Connors 1991; Vijayan and Kopell 2012). Their origins and functional role are still debated to this date and have been focused in several computational models (Bhattacharya et al. 2011; Jones et al. 2009; Vijayan and Kopell 2012). The presence of enhanced $\alpha$ activity may be interpreted either from a "processing" or "idling" standpoint and no categorical conclusion could be drawn regarding the metabolic origins of the observed increase in $\alpha$ power. A hypothesis is that it might be linked with perturbation of thalamo-cortical circuits and acute changes in thalamic hemodynamic activity consequent to awake surgery (Boyer et al. 2015a; Boyer et al. 2015b; Hughes and Crunelli 2005). Interestingly, Wijk et al. (2012) also found task-independant enhanced $\alpha$ rhythms for patients with glioma using a motor related paradigm consisting of self-paced opening and closing movement of the right or left hand (Wijk et al. 2012). Authors relied on physiological modelling and proposed that enhanced $\alpha$ activity may originate from the loss of feedback connections from other cortical regions. Neuronal oscillations above the $\theta$ range seemed to cover a large part of the occipital and parietal lobes and concentrate near ipsilesional perirolandic regions when progressing towards faster oscillations to the point that $\beta$ activity appeared as a focal point in the precentral gyrus, facing electrode $C 4 . \mu$ and $\beta$ waves near the central sulcus are often depicted as sensorimotor rhythms and have been involved in a large set of experimental paradigms including visual processing and stimulation, motor control, intention and imagery, or action anticipation (Arroyo et al. 1993; Denis et al. 2017; Han and Bin 2014; Heinrichs-Graham et al. 2017; Kim and Kim 2016; Sabate et al. 2012). But it is unknown whether the observed differences in faster activities were elicited by the experimental protocol itself or result from an intrinsic brain state, as healthy subjects did not show singular patterns of $\mu$ and $\beta$ oscillations in any experimental condition. $\mu$ waves are, for example, often proposed as a proxy for mirror neuron activity and their presence could be connected with postoperative recovery (Fox et al. 2016). $\beta$ activity, however, appeared to be stronger when the contralateral hand is recruited, which led to the conclusion that the observed alterations are, at least partially, task dependant and motor-related.

The present study had several limitations. the sample size was small because the postoperative assessments were lengthy and burdensome. Furthermore, the tumour size and site varied markedly from one patient to another. To circumvent these limitations, we used a differential approach in which individual performance levels were compared with a baseline. For each condition, each patient's performance was compared with the $95 \%$ CI derived from a control group. Differential approaches are particularly appropriate when between-subjects variability is large (as in many clinical studies) and so are fully justied in the present case. This approach was even more justified when the variability in the lesion extent and size is considered. It is important to bear in mind that lesion variability is always present in studies of patients with brain lesions. Even though researchers try to control for lesion size and locations, lesion reconstruction (when performed) is not usually accurate enough to determine which white matter pathways are injured, for instance. Furthermore, as demonstrated by functional mapping of the brain, there is also huge between-subjects variability in anatomical landmarks and functional performance. In many cases, datasets from individual patients are also averaged, constituting a major limitation. Given that white matter pathways link remote 
brain areas with various functions, small differences in the state of damage may lead to complete different functional states. Accordingly, we consider that our differential approach was justified for determining whether it was possible to measure changes in interhemispheric electrophysiological balance after awake brain surgery.

Overall, our results clearly identify changes in postoperative brainwaves of patients who underwent wide-awake surgery. Spectral analyses suggested imbalances between the injured and healthy hemispheres for patients. Interestingly, we observed that both $\delta$ and $\alpha$ activity appeared to be significantly higher over the whole scalp for the 3 patients at 3 months which could be a sign of the disruption of entire network(s) subsequent to surgery. Using fMRI, a recent study made a correspondence between resting state networks and brain oscillatory activity and stated that the dichotomy between the intrinsic vs. extrinsic systems (of networks) corresponds to the electrophysiological distinction between parts of the brain that communicate via $\theta$ vs. $\alpha$ phase synchrony. Thus, our results may indicate a perturbation of both systems for these patients (Hacker et al. 2017). These investigations performed on the motor preparation period also provided important information regarding longitudinal recovery of brain dynamics. Although all patients in our study had very different tumours, both in size and location, it's interesting to note that the 2 patients who underwent the experimental protocol respectively 9 and 12 months after surgery showed more moderate alterations of spectral content independently of the lesion size. This may be seen as an indicator for EEG signal standardization in time and presumably a resumption of brain dynamics. However, the fact that these patients still showed altered spectral power near the lesion site after such a long period may be indicative of a slower than expected recovery and/or may result from a new cortical configuration near the lesion site (new corticocortical junctions / axonal sprouting). These findings have potential clinical rehabilitation implications and suggest that a relatively simple motor task might be sufficient to monitor intraindividual brain dynamics recovery, using EEG measurement and a reduced number of features.

\section{Conflict of interest statement}

On behalf of all authors, the corresponding author states that there is no conflict of interest.

\section{Sponsorship and funding}

This work has been carried out with the support of the LabEx NUMEV project ( ${ }^{\circ}$ ANR-10LABX-20) funded by the French government's "Investissements d'Avenir" program, managed by the French National Research Agency (ANR) and complementary grants from the Institut Universitaire de France and INSERM laboratory (U1093).

\section{References}

1.Andersen, S M, S Z Rapcsak, and PM Beeson (2010). "Cost function masking during normalization of brains with focal lesions: still a necessity?” In: Neuroimage 53, pp. 78-84.

2. Arroyo, S, R P Lesser, B Gordon, S Uematsu, D Jackson, and R Webber (1993). 
"Functional significance of the mu rhythm of human cortex: an electrophysiologic study with subdural electrodes". In: Electroencephalogr Clin Neurophysiol 87, pp. 76-87.

3. Ashburner, J (2012). “SPM: A history”. In: Neuroimage 62, pp. 791-800.

4. Assenza, G, G Pellegrino, M Tombini, G Di Pino, and V Di Lazzaro (2015). "Wakefulness delta waves increase after cortical plasticity induction”. In: Clin Neurophysiol 126, pp. $1221-1227$.

5. Berger, H (1929). “On the electroencephalogram of man”. In: Arch Psychiatr Nervenkr 87, pp. 527-570.

6. Bhattacharya, B S, D Coyle, and LP Maguire (2011). "A thalamo-cortico-thalamic neural mass model to study alpha rhythms in Alzheimer's disease". In: Neural Netw 24, pp. 631-645.

7. Bonnetblanc, F, M Desmurget, and H Duffau (2006). "Low grade gliomas and cerebral plasticity: Fundamental and clinical implications". In: Medical Science 22, pp. 389-394.

8. Bonnetblanc, F, G Herbet, P Charras, M Hayashibe, D Guiraud, H Duffau, and B PoulinCharronnat (2014). "Asymmetric interhemispheric excitability evidenced by eventrelated potential amplitude patterns after "wide-awake surgery" of brain tumours". In: Exp Brain Res 232, pp. 3907-3918.

9. Boyer, A, J Deverdun, H Duffau, E Le Bars, N M Menjot de Champfleur, and F Bonnetblanc (2015a). "Crossed cerebellar diaschisis after awake brain surgery: Can we measure pre/post operative changes on resting state fMRI data?" In: 7th International IEEE/EMBS Conference on Neural Engineering 1, pp. 1032-1035.

10. Boyer, A, J Deverdun, H Duffau, E Le Bars, F Molino, N M Menjot de Champfleur, and F Bonnetblanc (2015b). "Longitudinal Changes in Cerebellar and Thalamic Spontaneous Neuronal Activity After Wide-Awake Surgery of Brain Tumors: a Resting-State fMRI Study". In: Cerebellum 15, pp. 451-465.

11. Brenner, R P, C F Reynolds, and RF Ulrich (1988). "Diagnostic efficacy of computerized spectral versus visual EEG analysis in elderly normal anddemented and depressed subjects". In: Electroenceph Clin Neurophysiol 69, pp. 110-117.

12. Brett, M, A P Leff, C Rorden, and J Ashburner (2001). "Spatial normalization of brain images with focal lesions using cost function masking”. In: Neuroimage 14, pp. 486500 .

13. Buchan, R J, K Nagata, E Yokoyama, P Langman, H Yuya, Y Hirata, J Hatazawa, and I Kanno (1997). "Regional correlations between the EEG and oxygen metabolism in dementia of Alzheimer's type". In: ElectroencephalogrClin Neurophysiol 1, pp. 409417.

14. Buzsaki, G and A Draguhn (2004). "Neuronal oscillations in cortical networks". In: Science 304, pp. 1926-1929.

15. Carmichael, S T and MF Chesselet (2002). "Synchronous neuronal activity is a signal for axonal sprouting after cortical lesions in the adult". In: J Neurosci 22, pp. 6062-6070.

16. Carpenter, J and J Bithell (2000). "Bootstrap confidence intervals: when, which, what? A practical guide for medical statisticians". In: Stat Med 19, pp. 1141-1164.

17. Charras, P, G Herbet, J Deverdun, N M de Champfleur, H Duffau, P Bartolomeo, and F Bonnetblanc (2014). "Functional reorganization of the attentional networks in lowgrade glioma patients: a longitudinal study". In: Cortex 63, pp. 27-41.

18. Claus, J J, V I Kwa, S Teunisse, G J Walstra, W A van Gool, J H Koelman, L J Bour, and BW Ongerboer de Visser (1998). "Slowing on quantitative spectral EEG is a marker for rate of subsequent cognitive and functional decline in early Alzheimer disease". In: Alzheimer Dis Assoc Disord 12, pp. 167-174.

19. Coben, L A, W Danziger, and M Storandt (1985). "A longitudinal EEG study of mild senile dementia of Alzheimer type: changes at 1 year and at 2 and 5 years". In: Electroencephalogr Clin Neurophysiol 61, pp. 101-112.

20. Decker, D A Jr and JR Knott (1972). "The EEG in intrinsic supratentorial brain tumors: a comparative evaluation”. In: Electroencephalogr Clin Neurophysiol 33, pp. 303-3010. 
21. Delorme, A and S Makeig (2004). "EEGLAB: an open source toolbox for analysis of single-trial EEG dynamics including independent component analysis". In: J Neurosci Methods 134, pp. 9-21.

22. Denis, D, R Rowe, A M Williams, and E Milne (2017). "The role of cortical sensorimotor oscillations in action anticipation". In: Neuroimage 146, pp. 1102-1114.

23. Desmurget, M, F Bonnetblanc, and H Duffau (2007). "Contrasting acute and slowgrowing lesions: A new door to brain plasticity”. In: Brain 130, pp. 898-914.

24. Duffau H (2015). "Stimulation mapping of white matter tracts to study brain functional connectivity". In: Nat Rev Neurol 11, pp. 255-265.

25. Duffau, H (2013). "Diffuse low-grade gliomas in adults: natural history, interaction with the brain, and new individualized therapeutic strategies". In: Springer London Ltd, pp. 601-602.

26. Duffau, H (2014a). "Diffuse low-grade gliomas and neuroplasticity". In: Diagn Interv Imaging 95, pp. 945-955.

27. Duffau, H (2014b). "The huge plastic potential of adult brain and the role of connectomics: new insights provided by serial mappings in glioma surgery". In: Cortex 58, pp. 325-337.

28. Duffau, H and L Capelle (2004). "Preferential brain locations of low-grade gliomas". In: Cancer 100, pp. 2622-2626.

29. Duffau, H, L Capelle, D Denvil, N Sichez, P Gatignol, M Lopes, M C Mitchell, J P Sichez, and R Van Effenterre (2003). "Functional recovery after surgical resection of low grade gliomas in eloquent brain: hypothesis of brain compensation”. In: J Neurol Neurosurg Psychiatry 74, pp. 901-907.

30. Foreman, B and J Claassen (2012). "Quantitative EEG for the detection of brain ischemia". In: Crit Care 16, p. 216.

31. Forstl, H, H Sattel, C Besthorn, S Daniel, C Geiger-Kabisch, F Hentschel, M Sarochan, and R Zerfass (1996). "Longitudinal cognitive, electroencephalographic and morphological brain changes in aging and Alzheimer's disease”. In: Br J Psychiatry 168, pp. 280-286.

32. Fox, N A, M J Bakermans-Kranenburg, K H Yoo, L C Bowman, E N Cannon, R E Vanderwert, P F Ferrari, and M H van IJzendoorn (2016). "Assessing human mirror activity with EEG mu rhythm: A meta-analysis”. In: Psychol Bull 142, pp. 291-313.

33. Garcia-Rill, E., S. D’Onofrio, B. Luster, S. Mahaffey, F. J. Urbano, and C. Phillips (2016). "The 10 Hz Frequency: A Fulcrum For Transitional Brain States". In: Transl Brain Rhythm 1, pp. 7-13.

34. Gueguen, B, D Ancri, C Derouesne, M C Bourdel, S Guillou, and E Landre (1991). "Comparison of SPECT and quantified EEG features in Alzheimer's type dementia". In: Neurophysiol Clin 21, pp. 377-387.

35. Hacker C D, A Z Snyder, M Pahwa M, M Corbetta, E C Leuthardt (2017). "Frequencyspecific electrophysiologic correlates of resting state fMRI networks". In: Neuroimage 149, pp. 446-457.

36. Han, Y and H Bin (2014). "Brain-Computer Interfaces Using Sensorimotor Rhythms: Current State and Future Perspectives". In: IEEE Trans Biomed Eng 61, pp. 1425-1435.

37. Harpale, V K and VK Bairagi (2016). "Time and frequency domain analysis of EEG signals for seizure detection: A review". In: IEEE MicroCom, DOI: 10.1109/MicroCom.2016.7522581.

38. Heinrichs-Graham, E, M J Kurz, J E Gehringer, and T W Wilson (2017). "The functional role of post-movement beta oscillations in motor termination". In: Brain Struct Funct 222, pp. 3075-3086.

39. Herbet, G, G Lafargue, F Bonnetblanc, S Moritz-Gassers, and H Duffau (2013). "Is the right frontal cortex really crucial in the mentalizing network? A longitudinal study in patients with a slow-growing lesion”. In: Cortex 49, pp. 2711-2727.

40. Herbet, G, M Maheu, E Costi, G Lafargue, and H Duffau (2016). "Mapping neuroplastic 
potential in brain-damaged patients". In: Brain 139, pp. 829-844.

41. Hughes, S W and V Crunelli (2005). "Thalamic mechanisms of EEG alpha rhythms and their pathological implications". In: Neuroscientist 11, pp. 357-372.

42. Jones, S R, D L Pritchett, M A Sikora, S M Stufflebeam, M Hamalainen, and CI Moore (2009). "Quantitative analysis and biophysically realistic neural modeling of the MEG mu rhythm: rhythmogenesis and modulation of sensory-evoked responses". In: $J$ Neurophysiol 102, pp. 3554-3572.

43. Jongh, A de, J C Baayen, J C de Munck, R M Heethaar, W P Vandertop, and CJ Stam (2003). "The influence of brain tumor treatment on pathological delta activity in MEG". In: Neuroimage 20, pp. 2291-2301.

44. Kim, J and S Kim (2016). "The effects of visual stimuli on EEG mu rhythms in healthy adults". In: J Phys Ther Sci 28, p. 1748.

45. Koberda, J L, A Moses, P Koberda, and L Koberda (2013). "Clinical advantages of quantitative electroencephalogram (QEEG)-electrical neuroimaging application in general neurology practice". In: Clin EEG Neurosci 44, pp. 273-285.

46. Mandonnet, E, P A Winkler, H Duffau (2010). "Direct electrical stimulation as an input gate into brain functional networks: principles, advantages and limitations." In: Acta Neurochir (Wien) 152, 185-193.

47. Medeiros Kanda, P A, R Anghinah, M T Smidth, and JM Silva (2009). "The clinical use of quantitative EEG in cognitive disorders". In: Dement neuropsychol 3.

48. Mellerio, C, M N Lapointe, P Roca, S Charron, L Legrand, J F Meder, C Oppenheim, and A Cachia (2016). "Identification of reliable sulcal patterns of the human Rolandic region". In: Front Hum Neurosci 10.

49. Morita, A, S Kamei, and T Mizutani (2011). "Relationship between slowing of the EEG and cognitive impairment in Parkinson disease". In: J Clin Neurophysiol 28, pp. 384387.

50. O'Gorman, R L, S S Poil, D Brandeis, P Klaver, S Bollmann, C Ghisleni, R Luchinger, E Martin, A Shankaranarayanan, D C Alsop, and L Michels (2013). "Coupling between resting cerebral perfusion and EEG”. In: Brain Topogr 26, pp. 442-457.

51. Palva, S and JM Palva (2007). "New vistas for alpha-frequency band oscillations". In: Trends Neurosci 30, pp. 150-158.

52. Pevzner, A, A Izadi, D J Lee, K Shahlaie, and GG Gurkoff (2016). "Making waves in the brain: what are oscillations and why modulating them makes sense for brain injury". In: Front Syst Neurosci 10, pp. 30.

53. Plaza, M, P Gatignol, M Leroy, and H Duffau (2009). "Speaking without Broca's area after tumor resection". In: Neurocase 15, pp. 294-310.

54. Preuß, M, S Preiss, S Syrbe, U Nestler, L Fischer, A Merkenschlager, A Bertsche, H Christiansen, and MK Bernhard (2015). "Signs and symptoms of pediatric brain tumors and diagnostic value of preoperative EEG”. In: Childs Nerv Syst 31, pp. 2051-2054.

55. Pritchard, W S, D W Duke, and KL Coburn (1991). "Altered EEG dynamical responsivity associated with normal aging and probable Alzheimer's disease”. In: Dementia 2, pp. 102-105.

56. Puthankattil Subha, D, P K Joseph, U Rajendra Acharya, and L Choo Min (2010). "EEG Signal Analysis: A Survey". In: J Med Syst 34, pp. 195-212.

57. Rabiller, G, J W He, Y Nishijima, A Wong, and J Liu (2015). "Perturbation of brain oscillations after ischemic stroke: a potential biomarker for post-stroke function and therapy". In: Int J Mol Sci 16, pp. 25605-25640.

58. Rorden, C, H O Karnath, and L Bonilha (2007). "Improving lesion-symptom mapping". In: J Cogn Neurosci 19, pp. 1081-1088.

59. Roschke, J and J Aldenhoff (1991). "The dimensionality of the human's electroencephalogram during sleep". In: Biol Cyber 64, pp. 307-313.

60. Sabate, M, C Llanos, E Enriquez, and M Rodriguez (2012). "Mu rhythm, visual processing and motor control”. In: Clin Neurophysiol 123, pp. 550-557. 
61. Sallard, E, H Duffau, and F Bonnetblanc (2012a). "Ultra-fast recovery from right neglect after 'awake surgery' for slow-growing tumor invading the left parietal area". In: Neurocase 18, pp. 80-90.

62. Sallard, E, J Barral, H Duffau, and F Bonnetblanc (2012b). "Manual reaction times and brain dynamics after 'awake surgery' of slow-growing tumours invading the parietal area. A case report". In: Brain Inj 26, pp. 1750-1755.

63. Schucht, P, S Moritz-Gasser, G Herbet, A Raabe, and H Duffau (2013). "Subcortical electrostimulation to identify network subserving motor control". In: Hum Brain Mapp 34, pp. 3023-3030.

64. Selvam, V S and S Shenbagadevi (2011). "Brain tumor detection using scalp eeg with modified Wavelet-ICA and multi layer feed forward neural network". In: Conf Proc IEEE Eng Med Biol Soc 1, pp. 6104-6109.

65. Selvam V S, S S Devi (2015). "Analysis of spectral features of EEG signal in brain tumor condition". In: Meas Sci Rev 15.

66. Silva, L R, Y Amitai, and BW Connors (1991). "Intrinsic oscillations of neocortex generated by layer 5 pyramidal neurons". In: Science 251, pp. 432-435.

67. Soininen, H, J Partanen, V Laulumaa, E L Helkala, M Laakso, and PJ Riekkinen (1991). "Longitudinal EEG spectral analysis in early stage of Alzheimer disease". In: Electroenceph Clin Neurophysiol 72, pp. 290-297.

68. Swisher, CB (2017). “QEEG in seizure detection”. In: Continuous EEG Monitoring, pp. 191-214.

69. Szelies, B, R Mielke, J Kessler, and WD Heiss (1997). "EEG power changes are related to regional cerebral glucose metabolism in vascular dementia". In: Clin Neurophysiol 110, pp. 615-620.

70. Tecchio, F, F Zappasodi, P Pasqualetti, M Tombini, C Salustri, A Oliviero, V Pizzella, F Vernieri, and P M Rossini (2005). "Rhythmic brain activity at rest from rolandic areas in acute mono-hemispheric stroke: a magnetoencephalographic study". In: Neuroimage 28, pp. 72-83.

71. Varela, F, J P Lachaux, E Rodriguez, and J Martinerie (2001). "The brainweb: phase synchronization and large-scale integration”. In: Nat Rev Neurosci 2, pp. 229-239.

72. Vijayan, S. and N.J. Kopell (2012). "Thalamic model of awake alpha oscillations and implications for stimulus processing”. In: Proc Natl Acad Sci U S A 109, pp. 1855318558.

73. Wijk, B C van, R B Willemse, W Peter Vandertop, and A Daffertshofer (2012). "Slowing of M1 oscillations in brain tumor patients in resting state and during movement". In: Clin Neurophysiol 123, pp. 2209-2212. 